DIFERENCIACIÓN EN LOS PRODUCTOS GASTRONÓMICOS: VENTAJAS PARA UN POSICIONAMIENTO EXITOSO

\title{
DIFERENCIACIÓN EN LOS PRODUCTOS GASTRONÓMICOS: \\ VENTAJAS PARA UN POSICIONAMIENTO EXITOSO
}

\author{
DIFFERENTIATION GASTRONOMIC PRODUCTS: \\ ADVANTAGES FOR A SUCCESSFUL POSITIONING
}
Diego Gustavo Ramón Molina*, Susana Suárez Reyes**, Guadalupe Janeth Cruz Luna***

\footnotetext{
*Licenciado en Mercadotecnia. Estudiante de Maestría en Administración y Dirección Estratégica. Universidad Juárez Autónoma de Tabasco.

**Licenciada en Administración. Estudiante de Maestría en Administración y Dirección Estratégica. Universidad Juárez Autónoma de Tabasco.

***Estudiante de la Licenciatura en Administración. Universidad Juárez Autónoma de Tabasco.
}

Dirección para recibir correspondencia: diego_gustavo_nike @hotmail.com 
DIFERENCIACIÓN EN LOS PRODUCTOS GASTRONÓMICOS: VENTAJAS PARA UN POSICIONAMIENTO EXITOSO

\section{RESUMEN}

OBJETIVO: Describir las ventajas que tiene realizar una estrategia de diferenciación de un producto gastronómico en general, basándonos en aspectos fundamentales del marketing como lo es el precio, producto, plaza o promoción.

MATERIAL Y MÉTODO: La metodología que se utilizó fue cuantitativa con enfoque descriptivo ya que lo que se buscaba era agotar las fuentes secundarias que afirmaran que la diferenciación es una clave para un buen posicionamiento en el ámbito del gastronómico.

RESULTADOS: En una serie de tablas se obtuvieron datos que muestran el comportamiento que tienen las personas a la hora de elegir un platillo, dejando en primer lugar que se dejan llevar por el sabor del mismo y dejando en segundo término el trato que le dan en el establecimiento.

CONCLUSIONES: Otro hallazgo que se pudo recabar de la investigación es que personas con un rango de edad entre 20 y 24 años son las que buscan experiencias de sabor culinarias, algo que debemos tener en claro es que el sector gastronómico está en total crecimiento, y la única manera de mantenerse a flote es con una diferenciación.

PALABRAS CLAVE: Gastronomía. Marketing. Posicionamiento. Diferenciación.

\section{ABSTRACT}

OBJECTIVE: Describe the advantages of making a differentiation strategy of a food product generally based on fundamental aspects of marketing such as the price, product, place and promotion.

MATERIAL AND METHOD: The methodology used was quantitative with descriptive approach since what the objective was exhausting secondary sources which affirm that differentiation is a key to a good position in the field of gastronomy.

RESULTS: In a series of tables data showing the behavior that people have when choosing a dish, leaving first that they are driven by the taste of it and leaving secondly the treatment they give in the facility they were obtained. 
DIFERENCIACIÓN EN LOS PRODUCTOS GASTRONÓMICOS: VENTAJAS PARA UN POSICIONAMIENTO EXITOSO

CONCLUSIONS: Another finding that could be collected from the research is that people within an age range from 20 to 24 years old are seeking new culinary taste experiences, something that we must be aware in the food sector because it is the only way to be outsatanding is with a differentiation.

KEYWORDS: Gastronomy. Marketing. Positioning. Differentiation.

\section{INTRODUCCIÓN}

\section{Historia de la gastronomía mexicana}

Según Elizalde, Martí y Martínez (2006), dice que alimentarse es una necesidad que todos los seres humanos tenemos de nacimiento y es tanto así, que Maslow en su pirámide hacia la autorealización propone como el nivel más básico a las necesidades fisiológicas (alimentarse, tomar agua,). Algo que nos distingue de los animales es el realizar nuestra propia comida, fue difícil en sus comienzos, pero con el descubrimiento del fuego, comenzaron a realizar comidas más sofisticadas creando así recetas que pasaron de generación en generación hasta llegar a nuestra actualidad (Ávila, Bastarrechea, Díaz, Flechsing, Moedano, Norman, Pérez, Salinas, Suarez y Vargas, 1988).

Las diferentes comidas conllevan ciertos aspectos, sabor u olor dependiendo de las diferentes especies que se utiliza, este varía según su región o cultura a la que pertenezcan (Solís y Estrada, 2014), según Lanz (2008), otros tantos de los platillos que se sirven en varios estados del país no cuentan con el mismo sabor, debido a la mezcla de tradición culinaria Prehispánica y Española, por eso desde entonces cada región creo una gastronomía propia teniendo así una cocina muy marcada en sabor, olor y consistencia.

Todas las regiones cuentan con alimentos únicos en su tipo, que reflejan su forma de cocinar, durante ciento de años los alimentos no solo satisfacían el paladar, sino que también era necesaria para nuestra salud y bienestar, ya que proporcionaban toda fuente de nutrientes (Pérez y Cisneros, s.f).

La Organización de las Naciones Unidas para la Educación, la Ciencia y la Cultura (UNESCO, 2010), en términos generales, expresó que la gastronomía de México es una de las más ricas a RAMÓN-MOLINA D. G., SUÁREZ-REYES S., CRUZ-LUNA G. J. 
DIFERENCIACIÓN EN LOS PRODUCTOS GASTRONÓMICOS: VENTAJAS PARA UN POSICIONAMIENTO EXITOSO

nivel mundial, ya que declaró a la cocina Mexicana, en específico a la cocina tradicional, como "patrimonio cultural inmaterial de la humanidad", debido a que muchas personas y restaurantes aún siguen utilizando recetas y técnicas de nuestros antepasados, por otra parte Perales y Gonzales (2013) mencionan que, algunas técnicas han evolucionado a la par de la vida cotidiana de nuestra sociedad, debido a este ritmo tan acelerado, queda menos tiempo a las personas para cocinar.

Al igual que la evolución de la sociedad, el arte culinario tuvo que hacerlo, alcanzado parámetros inimaginables, ya no se requiere solo saber cocinar si no que necesita pasión, creatividad y dedicación, para poder satisfacer los gustos del consumidor (Muñoz, Tamayo y Hernández, 2012).

La gastronomía es un factor importante para la economía, cuenta con 2 tipos de consumidores, el local que es toda la población que se encuentra en una determinada región y el sector turístico, son personas que llegan esporádicamente a alguna región y consumen cierto platillo (Pérez y Cisneros, s.f) y lo sustenta la Secretaría de Turismo (SECTUR, 2015), ya que menciona que la comida es una de los principales gastos que tienen los turistas al visitar tierras mexicanas, se calculó que en el 2015 el sector gastronómico aporto más de 1.3 millones de empleo directo.

Debido a eso el sector gastronómico ha crecido en un área de mucha competencia donde se desarrollan negocios todos los días, con mejores estrategias, servicios y sobre todo sabe diferenciar sus productos de los demás, en otras palabras, utilizan mejor marketing (Cabrera, 2013).

\section{Aspectos del marketing para una estrategia}

En Latinoamérica, se usan palabras como, mercadeo, comercialización, diferenciación y posicionamiento, pero todos estos términos son parte de la Mercadotecnia, aunque muchos no conocen los términos y suelen creer que el marketing es solo ventas, publicidad, investigación de mercados (Carasila, 2008). Según Kotler y Armstrong (2012) menciona que en su versión más simple de lo que es marketing lo describen como "la administración de relaciones redituables con el cliente", es decir hay que tener en cuenta o en otros términos estar al pendiente del cliente, en todas sus necesidades y cambios que pueda tener.

Por otro lado, Monferrer (2013) apoya la teoría de Carasila (2008) donde afirma que el marketing no es sinónimo de publicidad y ventas si no que más bien son funciones de la mercadotecnia, RAMÓN-MOLINA D. G., SUÁREZ-REYES S., CRUZ-LUNA G. J. 


\section{DIFERENCIACIÓN EN LOS PRODUCTOS GASTRONÓMICOS: VENTAJAS PARA UN} POSICIONAMIENTO EXITOSO

también afirma que otra de las funciones marketing es influenciar al consumidor a comprar determinado producto, aunque ellos no tengan ninguna intención de hacerlo.

Es por eso que marketing lo podemos definir como el proceso social, que tiene como objetivo ayudar a ambas partes, tanto al consumidor, como al vendedor; satisfaciendo sus necesidades por parte del vendedor y pagándole con incentivos económicos por parte del consumidor (Gómez, 2004).

Para que el marketing pueda atender cualquier mercado en cualquier sector ya sea, gastronómico, industrial, social, etc. Debe considerar una serie de factores que los expertos le llaman la mezcla del marketing, marketing mix o como usualmente se le conoce como las 4’ $p$, que son: Precio, Plaza, Promoción y Producto (Hernández y Maubert, 2009).

\section{Tabla 1}

Componentes del marketing mix

\begin{tabular}{|c|c|c|}
\hline Marketing mix & Características & Descripción \\
\hline Producto & $\begin{array}{l}\text { Variedad de productos, } \\
\text { calidad, diseño, marca, } \\
\text { características, tamaños, } \\
\text { servicios y garantía }\end{array}$ & $\begin{array}{l}\text { Es todo lo que pueda ser vendido, } \\
\text { pueden ser tangible o intangibles, por } \\
\text { ejemplo, lavadoras, licuadoras, comidas, } \\
\text { así como auto-lavados, asesorías, } \\
\text { peluquerías (Peñaloza, 2005) y (Acuña, } \\
\text { 2013). }\end{array}$ \\
\hline Plaza & $\begin{array}{l}\text { - Canales - } \text { Cobertura } \\
\text { - Ubicación - Inventario } \\
\text { - Transporte }\end{array}$ & $\begin{array}{l}\text { La plaza se refiere al lugar en donde los } \\
\text { consumidores pueden obtener el } \\
\text { producto; es decir, su punto de venta } \\
\text { (Peñaloza, 2005) y (Acuña, 2013). }\end{array}$ \\
\hline Precio & $\begin{array}{l}\text { Lista de precios } \\
\text { - Descuentos - Concesiones } \\
\text { - Periodos de pago } \\
\text { - Condiciones de crédito }\end{array}$ & $\begin{array}{l}\text { Este es de suma importancia ya que con } \\
\text { ello se puede seguir con el correcto } \\
\text { funcionamiento de la empresa, el precio } \\
\text { es el cambio en efectivo por algún bien o } \\
\text { servicio (Peñaloza, 2005) y (Acuña, } \\
\text { 2013). }\end{array}$ \\
\hline Promoción & $\begin{array}{l}\text { - Promoción y ventas } \\
\text { - Anuncios - Fuerza de } \\
\text { ventas - Relaciones públicas } \\
\text { - Marketing directo }\end{array}$ & $\begin{array}{l}\text { Es una estrategia muy utilizada por las } \\
\text { empresas para conseguir clientes o bien } \\
\text { poder tener una fuerte demanda de sus } \\
\text { productos con ciertos incentivos; es } \\
\text { decir, descuentos (Peñaloza, 2005) y } \\
\text { (Acuña, 2013). }\end{array}$ \\
\hline
\end{tabular}

Fuente: Elaboración propia basado en Álvarez (2007), Peñaloza (2005) y Acuña (2013). 


\section{DIFERENCIACIÓN EN LOS PRODUCTOS GASTRONÓMICOS: VENTAJAS PARA UN} POSICIONAMIENTO EXITOSO

El marketing mix es una de las tantas herramientas que ofrece la mercadotecnia, esta herramienta ayuda a conocer la situación de la empresa y poder desarrollar una estrategia específica de posicionamiento (Álvarez, 2007).

Estamos inmersos en la globalización lo que hace que nuestra permanencia en el mundo de los negocios se vuelva más competitivo, haciendo el posicionamiento un método más sofisticado con estrategias más elaboradas (Torres, 2012).

La diferenciación, en un mercado donde hay mucho de lo mismo, seguir vendiendo de la misma manera no es aceptable, diferenciarte es especializarte en un nicho de mercado en concreto para ello existen dos tipos de diferenciación, la horizontal y la vertical (Vela, 2012).

Según Sandoval (2005), menciona que la diferenciación vertical se enfoca en la diferenciación por como lo ofreces, es decir puede consistir en el tipo de promoción, precio o servicio que puedas manejar, mientras que la diferenciación horizontal se enfoca en la diferenciación por lo que ofreces; es decir, la gran variedad de productos que puedas ofrecer o en la satisfacción que le puedas dejar, como lo afirma Cardozo, Hernandis y Ramírez (2014), la diferenciación es llenar de características diferentes a ciertos productos ya sea de calidad, estilo o imagen de marca, pero que el cliente siempre pueda percibir las nuevas características.

\section{Tabla 2}

\section{Formas de diferenciación en la gastronomía}

\begin{tabular}{|c|c|c|}
\hline Marketing mix & Diferenciación & Ventajas \\
\hline Precio & $\begin{array}{l}\text { Según Espinoza (2014) y Thompson, } \\
\text { Peteraf, Gamble y Strickland (2012 } \\
\text { p.142) diferenciarnos por precio no es } \\
\text { nada fácil, ya que resulta un arma de } \\
\text { doble filo, subir el precio valora más mi } \\
\text { marca, pero de igual manera puede ser } \\
\text { que tenga menos compradores. }\end{array}$ & $\begin{array}{l}\text {-Si la gastronomía que se vende es de calidad, te puedes } \\
\text { dar el lujo de tener precios un poco elevado, sabiendo que } \\
\text { las personas adquirirán el platillo (Capetillo, 2013). }\end{array}$ \\
\hline Plaza & $\begin{array}{l}\text { Este se puede diferenciar según la } \\
\text { ubicación, su espacio, comodidad o } \\
\text { simplemente visibilidad (Verdú, 2000). }\end{array}$ & $\begin{array}{l}\text { Hay que tener en claro que el lugar, tiene que ir acorde a } \\
\text { los platillos típicos que se vendan, esto dará mayor } \\
\text { impresión y fidelidad por parte de los clientes (Capetillo, } \\
\text { 2013). }\end{array}$ \\
\hline Producto & $\begin{array}{l}\text { Según Álvarez (2007) y Espinoza } \\
\text { (2014) Un producto puede ser } \\
\text { diferenciado por la imagen, la marca, } \\
\text { características o diseño. }\end{array}$ & $\begin{array}{l}\text {-Tu receta es única y el sabor difícilmente podrá ser } \\
\text { copiado por otras personas (Capetillo, 2013). }\end{array}$ \\
\hline Promoción & $\begin{array}{l}\text { Según Álvarez (2007) una forma de } \\
\text { diferenciarse es mediante la } \\
\text { promoción, ya sea porque repartes } \\
\text { cupones, haces descuentos o tus } \\
\text { anuncios son llamativos. }\end{array}$ & $\begin{array}{l}\text { Al tener una marca y con la promoción adecuada, podrán } \\
\text { captar mayores clientes, es decir más gente conocerá los } \\
\text { platillos que venda (Capetillo, 2013). }\end{array}$ \\
\hline
\end{tabular}

Fuente: Elaboración propia basado en Espinoza (2014), Thompson, Peteraf, Gamble y Strickland (2012 p.142), Álvarez (2007), Verdú (2000) y Capetillo (2013).

RAMÓN-MOLINA D. G., SUÁREZ-REYES S., CRUZ-LUNA G. J. 
DIFERENCIACIÓN EN LOS PRODUCTOS GASTRONÓMICOS: VENTAJAS PARA UN POSICIONAMIENTO EXITOSO

Teniendo en cuenta las 4’ p del marketing y la estrategia de diferenciación, se entiende con mayor claridad cómo se puede posicionar un proveedor de servicios en la mente del consumidor, y si se fusionaran estas dos poderosas estrategias, crearían unos excelentes resultados (Cabrera, 2013).

\section{MATERIAL Y MÉTODO}

La metodología que se utilizó fue cuantitativa con enfoque descriptivo (Hernández, Fernández y Baptista, 2010), ya que lo que se buscaba era agotar las fuentes secundarias que afirmaran que la diferenciación es una clave para un buen posicionamiento en el ámbito del gastronómico. Cabe mencionar, que la investigación fue apoyada con un sondeo, la cual se realizó a través de diversas redes sociales y plataformas web, de igual manera fue realizado a 89 personas de entre un rango de edad de 20 a 34 años; lo que se busca con esto es, obtener información sobre qué es lo primero en lo que piensan al adquirir un producto gastronómico.

\section{Muestra}

La muestra que se utilizó en el presente trabajo fueron personas residentes del estado de Tabasco, específicamente personas de entre 20 y 34 años, a las cuales se realizó un sondeo exprés, con la finalidad de determinar que es en lo primero que piensan al elegir un platillo, el tipo de muestra fue no probabilística, por lo cual se le aplicó el sondeo a 89 personas al azar, que cumpliera con el requisito previamente mencionado.

\section{Técnica de investigación}

La técnica empleada para la presente investigación fue la investigación en fuentes secundarias, apoyada de un sondeo, puesto que se requería recolectar información sobre el pensamiento que tienen los tabasqueños a la hora de elegir un platillo gastronómico.

\section{Instrumento}

Para la recolección de datos, se realizó un sondeo en el cual se solicitó información a través de las siguientes variables:

- Género.

- Edad.

- Motivación para elegir un platillo gastronómico (precio, servicio, sabor e imagen). RAMÓN-MOLINA D. G., SUÁREZ-REYES S., CRUZ-LUNA G. J. 
DIFERENCIACIÓN EN LOS PRODUCTOS GASTRONÓMICOS: VENTAJAS PARA UN POSICIONAMIENTO EXITOSO

\section{RESULTADOS}

\section{Tabla 3}

Distribución de frecuencias

\begin{tabular}{lcccc}
\hline & & Frecuencia & $\%$ & \% válido \\
\hline ¿A la hora de elegir un & El servicio & 25 & 28.1 & 28.1 \\
producto gastronómico & El precio & 15 & 16.9 & 16.9 \\
(específicamente & El sabor & 37 & 41.6 & 41.6 \\
comida) en que es lo & La imagen del platillo & 12 & 13.5 & 13.5 \\
primero que piensas? & Total & 89 & 100.0 & 100.0 \\
\hline
\end{tabular}

Fuente: Elaboración propia.

En la tabla 3, puede observarse que el $41.6 \%$ de los encuestados sostuvo que, a la hora de elegir un producto gastronómico, se van más por el sabor, dejando en segundo lugar al servicio con $28.1 \%$, en tercer lugar, el precio con solo $16.9 \%$; y por último, la imagen del platillo con tan solo el $13.5 \%$ de los encuestado. Dejando en claro con los datos previamente descritos, que del marketing mix se van más hacia el producto y de los tipos de diferenciación a los proveedores de servicios les conviene más la diferenciación vertical.

\section{Tabla 4}

Distribución de frecuencias por edad

\begin{tabular}{|c|c|c|c|c|c|c|}
\hline & & & \multicolumn{3}{|c|}{ Edad } & \multirow[t]{2}{*}{ Total } \\
\hline & & & $20-24$ & $25-29$ & $30-34$ & \\
\hline \multirow{8}{*}{$\begin{array}{l}\text { ¿A la hora de elegir } \\
\text { un producto } \\
\text { gastronómico } \\
\text { (específicamente } \\
\text { comida) en que es } \\
\text { lo primero que } \\
\text { piensas? }\end{array}$} & \multirow[t]{2}{*}{ El servicio } & $\mathrm{n}$ & 19 & 4 & 2 & 25 \\
\hline & & $\%$ & $33.3 \%$ & $18.2 \%$ & $20.0 \%$ & $28.1 \%$ \\
\hline & \multirow[t]{2}{*}{ El precio } & $\mathrm{n}$ & 8 & 6 & 1 & 15 \\
\hline & & $\%$ & $14.0 \%$ & $27.3 \%$ & $10.0 \%$ & $16.9 \%$ \\
\hline & \multirow[t]{2}{*}{ El sabor } & $\mathrm{n}$ & 22 & 8 & 7 & 37 \\
\hline & & $\%$ & $38.6 \%$ & $36.4 \%$ & $70.0 \%$ & $41.6 \%$ \\
\hline & \multirow[t]{2}{*}{ La imagen del platillo } & $\mathrm{n}$ & 8 & 4 & 0 & 12 \\
\hline & & $\%$ & $14.0 \%$ & $18.2 \%$ & $0.0 \%$ & $13.5 \%$ \\
\hline \multirow{2}{*}{\multicolumn{2}{|c|}{ Total }} & $\mathrm{n}$ & 57 & 22 & 10 & 89 \\
\hline & & $\%$ & $100.0 \%$ & $100.0 \%$ & $100.0 \%$ & $100.0 \%$ \\
\hline
\end{tabular}

Fuente: Elaboración propia. 
DIFERENCIACIÓN EN LOS PRODUCTOS GASTRONÓMICOS: VENTAJAS PARA UN POSICIONAMIENTO EXITOSO

En la tabla 4, se tiene la distribución de frecuencias por el rango de edad, lo que indica que la mayor población se declina por el sabor, teniendo como resultado que el $38.6 \%$ de los encuestados tienen un rango de edad de 20 a 24 , el $36.4 \%$ de los encuestados tienen un rango de edad de entre 25 y 29 años; y por último, de las personas que fueron encuestadas de un rango de edad entre 30 y 34 años el 70\% eligió el sabor.

\section{Tabla 5}

Distribución de frecuencias por género

\begin{tabular}{|c|c|c|c|c|c|}
\hline & & & \multicolumn{2}{|c|}{ Genero } & \multirow[t]{2}{*}{ Total } \\
\hline & & & Hombre & Mujer & \\
\hline \multirow{8}{*}{$\begin{array}{l}\text { ¿A la hora de elegir un } \\
\text { producto gastronómico } \\
\text { (específicamente comida) } \\
\text { en que es lo primero que }\end{array}$} & El servicio & $n$ & 9 & 16 & 25 \\
\hline & & $\%$ & $29.0 \%$ & $27.6 \%$ & $28.1 \%$ \\
\hline & El precio & $\mathrm{n}$ & 10 & 5 & 15 \\
\hline & & $\%$ & $32.3 \%$ & $8.6 \%$ & $16.9 \%$ \\
\hline & El sabor & $\mathrm{n}$ & 12 & 25 & 37 \\
\hline & & $\%$ & $38.7 \%$ & $43.1 \%$ & $41.6 \%$ \\
\hline & La imagen del & $\mathrm{n}$ & 0 & 12 & 12 \\
\hline & platillo & $\%$ & $0.0 \%$ & $20.7 \%$ & $13.5 \%$ \\
\hline \multirow{2}{*}{\multicolumn{2}{|c|}{ Total }} & $\mathrm{n}$ & 31 & 58 & 89 \\
\hline & & $\%$ & $100 \%$ & $100 \%$ & $100 \%$ \\
\hline
\end{tabular}

Fuente: Elaboración propia.

En la tabla 5, se observa la distribución de frecuencias por género, y constata algo muy peculiar que el $32 \%$ de los que declinaron por el precio son hombres; es decir, 10 de 31 hombres encuestados se preocupan por el dinero, mientras que solo 5 mujeres de 58 se preocupan por lo mismo. Un dato curioso que dio a conocer el sondeo, es que solo 12 mujeres de 58 se preocupan por la esteticidad del platillo, y de igual manera mostrando que ningún hombre contestó positivo esta variable, existiendo un nicho de mercado solo para mujeres, enfocándose en realizar platillos estéticos o quizás personalizados. 
DIFERENCIACIÓN EN LOS PRODUCTOS GASTRONÓMICOS: VENTAJAS PARA UN POSICIONAMIENTO EXITOSO

\section{CONCLUSIONES}

Queda claro que el sector gastronómico está en total crecimiento, y la única manera de mantenerse a flote es con una diferenciación; es decir, utilizar el marketing con todos sus elementos (Cabrera, 2013). Hoy en día, se necesita más que procesos administrativos, se necesita tener más de visión; por ejemplo, elaborar estrategia por todos los medios disponibles que permitirá diferenciar nuestro producto o servicio ante el consumidor (Torres, 2012).

Dejando a un lado cualquier estrategia que más le convenga, no se puede negar que el negocio de la gastronomía puede fortalecer la crisis económica de las familias mexicanas, y si se añade que la gastronomía sea con platillos típicos de su región, esto hace que se vuelva más atractiva, tanto para los consumidores extranjeros como para los locales (Pérez y Cisneros, s.f).

\section{REFERENCIAS BIBLIOGRÁFICAS}

Acuña, J. A. (17 de mayo 2013). Las 4 P (precio, producto, plaza y promoción). Overblog, [comentario de un blog]. Recuperado de http://acualonit.overblog.com/las-4-p-preciproducto-plaza-y-promocion

Álvarez, D. (2007). Planificación estratégica de marketing. Perspectivas, pp. 67-104. Recuperado de http://www.redalyc.org/articulo.oa?id=425942331006

Ávila, D., Bastarrechea, R., Díaz, A., Flechsing, G., Moedano, G., Norman, O., Pérez, G., Salinas, G., Suarez, C., y Vargas, L. (1988). Atlas cultura de México. Editorial Planeta. Recuperado de https://professionalchefenriquez.files.wordpress.com/2011/08/atlas-culturalgastronomia.pdf.

Cabrera, S. (2013). Marketing gastronómico. La experiencia de convertir el momento del consumo en un recuerdo memorable. Centro de Estudios en Diseño y Comunicación, p 165-174. Recuperado de http://fido.palermo.edu/servicios_dyc/publicacionesdc/archivos/421_libro.pdf

Capetillo, V. (16 de mayo 2013). El mix de marketing de un restaurante de cocina de autor. Marketing de restaurantes. [Comentario de un blog]. Recuperado de http://marketingderestaurantes.com/TipsRestaurantes/el-mix-de-marketing-de-unrestaurante-de-cocina-de-autor/

Carasila, A. (2008). El concepto de Marketing: pasado y presente. Revista de Ciencias Sociales (Ve), XIV, pp. 391-412. Recuperado de http://www.redalyc.org/articulo.oa?id=28011672014 RAMÓN-MOLINA D. G., SUÁREZ-REYES S., CRUZ-LUNA G. J. 
DIFERENCIACIÓN EN LOS PRODUCTOS GASTRONÓMICOS: VENTAJAS PARA UN POSICIONAMIENTO EXITOSO

Cardozo, J., Hernandis, B. y Ramírez, N. (2014). Caracterización de los sistemas de productos en el marco de la personalización, la variabilidad y la diferenciación. Un estudio con expertos. Revista chilena de ingeniería, 22(2), 2014, pp. 278-291. Recuperado de http://www.scielo.cl/scielo.php?script=sci_arttext\&pid=S0718-33052014000200013

Elizalde, A., Martí, M. y Martínez, A. (2006). Una revisión crítica del debate sobre las necesidades humanas desde el Enfoque Centrado en la Persona. POLIS. Revista Latinoamericana, 5. Recuperado de http://www.redalyc.org/articulo.oa?id=30517306006

Espinoza, R. (6 mayo 2014). Estrategia de marketing. [Comentario de un blog]. Recuperado de http://robertoespinosa.es/2014/05/06/marketing-mix-las-4ps-2/

Gómez, H. (2004). Marketing de emociones. La forma para lograr fidelidad de los clientes. Semestre Económico, 7, pp. 203-211. Recuperado de http://www.redalyc.org/articulo.oa?id=165013657008

Hernández, C. y Maubert, C. (2009). Fundamentos de marketing. México: Pearson Educación. Kotler, P. y Armstrong, G. (2012). Marketing. México: Pearson Educación.

Lanz, M. (2008). La gastronomía en el contexto de la cultura mexicana. Colección: Instituto Campechano No. 6 recuperado de http://instcamp.edu.mx/investigacioneshistoricas/wpcontent/uploads/2016/10/06-La-Gastronomia.pdf

Monferrer, D. (2013). Fundamentos de marketing. Universitat jaune DOI: http://dx.doi.org/10.6035/Sapientia74

Muñoz, I., Tamayo, A. y Hernández, C. (2012). Formación profesional de la gastronomía en instituciones públicas en México. Revista Electrónica "Actualidades Investigativas en Educación", $\quad 12, \quad$ pp. 1-18. $\quad$ Recuperado de http://www.redalyc.org/articulo.oa?id=44723985008

Navarro, M. (2012). Técnicas de ventas. Red tercer milenio.

Perales, H y Gonzales, B. (2013). Alimentos mexicanos empaquetados, envasados y enlatados: tradición lista para servir. Revista del consumidor. Recuperado de http://www.gob.mx/cms/uploads/attachment/file/100471/RC439_Lab_Prof_Alimentos_Mexi canos.pdf

Pérez, M. y Cisneros, W. (s.f). El lado cultural del patrimonio gastronómico mexicano. Recuperado de http://web.uaemex.mx/Culinaria/tres_ne/articulo_06.pdf 
DIFERENCIACIÓN EN LOS PRODUCTOS GASTRONÓMICOS: VENTAJAS PARA UN POSICIONAMIENTO EXITOSO

Peñaloza, M. (2005). El mix de marketing: Una herramienta para servir al cliente. Actualidad Contable Faces, 8, pp. 71-81. Recuperado de http://www.redalyc.org/articulo.oa?id=25701007

Sandoval, A. (2005). La diferenciación de productos: Una breve nota. Entramado, 1, pp. 34-47. Recuperado de http://www.redalyc.org/articulo.oa?id=265420471005

Secretaría de turismo [SECTUR]. (31 DE MAYO 2015). Boletín 98.- Gastronomía Mexicana, con el potencial para ser la mejor del mundo: SECTUR. [Comentario de un blog]. Recuperado de http://www.sectur.gob.mx/sala-de-prensa/2015/05/31/boletin-98-gastronomiamexicana-con-el-potencial-para-ser-la-mejor-del-mundo-sectur/

Solís, C. y Estrada, E. (2014). Prácticas culinarias y (re)conocimiento de la diversidad local de verduras silvestres en el Colectivo Mujeres y Maíz de Teopisca, Chiapas, México. Recuperado de http://www.scielo.org.mx/scielo.php?script=sci_arttext\&pid=S1665$80272014000200010 \&$ lang $=p t$

Thompson, A. A., Peteraf, M. A., Gamble, J. E. y Strickland, A. J. (2012). Administración Estratégica. Teoría y casos. México: McGraw-Hill

Torres, A. (2012). El Posicionamiento la diferenciación estrategias eficaces en el mercado competitivo. Recuperado de http://biblioteca.unitecnologica.edu.co/notas/tesis/0064096.pdf

Organización de las naciones unidas para la educación, la ciencia y la cultura [UNESCO] (junio 2010). La cocina tradicional mexicana, cultura comunitaria, ancestral y viva - El paradigma de Michoacán. [Comentario en un blog]. Recuperado de https://ich.unesco.org/es/RL/lacocina-tradicional-mexicana-cultura-comunitaria-ancestral-y-viva-el-paradigma-demichoacan-00400

Vela, L. (2012). Diferenciación de productos. Universidad Nacional de Perú. Recuperado de https://web.ua.es/es/giecryal/documentos/diferenciacionproductos.pdf?noCache $=1354884406288$

Verdu, F. (2000). Estrategias de diferenciación y tamaño de empresa un análisis empírico aplicado a los sectores industriales de la comunidad valenciana. Investigaciones Europeas de Dirección y Economía de la Empresa. 6, pp. 131-158 Recuperado de http://redaedem.org/ar 